\section{Dynamic regulatory interactions of Polycomb group genes: $M E D E A$ autoregulation is required for imprinted gene expression in Arabidopsis}

\author{
Célia Baroux, ${ }^{1}$ Valeria Gagliardini, ${ }^{1}$ Damian \\ R. Page, ${ }^{2}$ and Ueli Grossniklaus ${ }^{3}$ \\ Institute of Plant Biology and Zürich-Basel Plant Science \\ Center, University of Zürich, CH-8008 Zürich, Switzerland
}

The imprinted Arabidopsis Polycomb group (PcG) gene MEDEA (MEA), which is homologous to Enhancer of Zeste $[E(Z)]$, is maternally required for normal seed development. Here we show that, unlike known mammalian imprinted genes, MEA regulates its own imprinted expression: It down-regulates the maternal allele around fertilization and maintains the paternal allele silent later during seed development. Autorepression of the maternal $M E A$ allele is direct and independent of the MEA-FIE (FERTILIZATION-INDEPENDENT ENDOSPERM) PcG complex, which is similar to the $\mathrm{E}(\mathrm{Z})$-ESC (Extra sex combs) complex of animals, suggesting a novel mechanism. A complex network of cross-regulatory interactions among the other known members of the MEA-FIE PcG complex implies distinct functions that are dynamically regulated during reproduction.

Supplemental material is available at http://www.genesdev.org.

Received December 29, 2005; revised version accepted February 23, 2006.

Polycomb group (PcG) genes play an essential role during development of multicellular organisms. PcG genes were first identified in Drosophila as mutants causing homeotic transformations because they lead to a deregulation of the HOX genes. While an early cascade of transcription factors establishes specific HOX gene expression patterns, PcG proteins act in multimeric complexes to maintain the repressed state of their target genes throughout development (Ringrose and Paro 2004). Two distinct PcG complexes have been described in animals, the Polycomb Repressive Complex 1 (PRC1) and the Enhancer of Zeste-Extra sex combs [E(Z)-ESC] complex (also known as PRC2). These complexes consist of several core components but associate with additional proteins to form distinct subcomplexes in specific tissues (Otte and Kwaks 2003).

[Keywords: Arabidopsis; genomic imprinting; MEDEA; Polycomb group; regulation; seed development]

${ }^{1}$ These authors contributed equally to this work.

${ }^{2}$ Present address: Institut Pasteur, Unité de Génétique Moléculaire $\mathrm{Mu}$ rine, 25 rue du Docteur Roux, 75015 Paris, France.

${ }^{3}$ Corresponding author.

E-MAIL, grossnik@botinst.unizh.ch; FAX 41-(0)44-634-82-04.

Article and publication are at http://www.genesdev.org/cgi/doi/10.1101/ gad.378106.
PcG proteins are structurally heterogenous, but some show functional and structural conservation among Drosophila, mammals, and higher plants (Reyes and Grossniklaus 2003; Ringrose and Paro 2004). While no homologs of PRCl components have been found in the Arabidopsis genome, the core components of the E(Z)ESC complex are conserved. There are several variants of the E(Z)-ESC complex in plants (Reyes and Grossniklaus 2003; Chanvivattana et al. 2004), the best studied being the MEA-FIE complex (Köhler et al. 2003a), which regulates cell proliferation during reproductive development. It consists of the FIS class PcG proteins MEDEA (MEA), FERTILIZATION-INDEPENDENT ENDOSPERM (FIE), and FERTILIZATION-INDEPENDENT SEED2 (FIS2), homologs of the Drosophila E(Z), ESC, and Suppressor of zeste12 [SU(Z)12], respectively (Grossniklaus et al. 1998; Luo et al. 1999; Ohad et al. 1999). In addition, animal and plant complexes contain homologs of Drosophila p55, a histone-binding WD40 repeat protein (in Arabidopsis known as MSI1) (Hennig et al. 2003), present in several chromatin-associated complexes.

The Arabidopsis MEA-FIE complex plays an essential role in sexual reproduction. In flowering plants, meiotic products do not directly differentiate into gametes. Rather, they form multicellular structures, the gametophytes, producing the gametes. The male gametophyte, or pollen, carries two sperm cells that it delivers to the ovule. The female gametophyte, or embryo sac, is enclosed in the ovule and carries the egg and the central cell, which fuse with one sperm each to form the embryo and endosperm, respectively. Mutations impairing components of the MEA-FIE complex show pre- and postfertilization phenotypes associated with abnormal cell proliferation (Köhler and Grossniklaus 2002; Guitton and Berger 2005). In these fis class mutants (mea, fie, fis 2 , and msi1), the central cell initiates endosperm development without fertilization. If fertilized, embryo and endosperm derived from fis mutant gametes show defects in the regulation of cell proliferation. These phenotypes indicate that the Arabidopsis MEA-FIE complex shares functional similarities with the corresponding mammalian complex, where mutations in genes encoding its subunits cause aberrant cell proliferation and cancer (Valk-Lingbeek et al. 2004).

Importantly, in FIS class genes all mutations lead to maternal-effect seed abortion. For $M E A$, it was shown that the maternal effect is due to the regulation of $M E A$ by genomic imprinting. $M E A$ is activated prior to fertilization in the embryo sac, and the activity of the maternally inherited allele is maintained in the developing embryo and endosperm, whereas the paternally inherited allele is silent (Kinoshita et al. 1999; Vielle-Calzada et al. 1999). The molecular mechanisms controlling imprinted gene expression at the $M E A$ locus are not fully elucidated. Two regulators have been identified that control maternal activation of $M E A$ prior to fertilization. DEMETER (DME), a DNA-glycosylase, is thought to remove methylated cytosines at the MEA locus and acts antagonistically to the DNA-methyltransferase MET1 (Xiao et al. 2003; Choi et al. 2004). However, while there is genetic evidence for an involvement of DME and MET1 in the regulation of $M E A$, it is not known whether these are direct or indirect regulators. 
Here we report that MEA is involved in the regulation of its own imprinted expression, a feature that has not been described for imprinted genes in animals. Before and after fertilization, MEA is required to repress expression of the maternal $M E A^{m}$ allele, while later in seed development, MEA keeps the paternally inherited $M E A^{p}$ allele silent. Around fertilization MEA interacts with its own promoter, as shown by chromatin immunoprecipitation (ChIP) experiments, suggesting that MEA directly controls transcriptional repression of the $M E A^{m}$ allele. Importantly, this autoregulatory function of MEA is independent of the MEA-FIE complex since MEA expression is not altered in other fis class mutants. This reveals a novel, MEA-FIE complex-independent function of MEA, so far not described for its animal counterpart. We investigated whether other members of the MEA-FIE complex autoregulate and found a highly dynamic crossregulatory network. Surprisingly, the $P c G$ genes were involved in both negative and positive regulation, suggesting that plant PcG proteins may share dual repression/ activation functions, directly or indirectly. This indicates that, as its animal counterpart, the formation of the MEA-FIE complex and related subcomplexes is highly dynamic during plant reproduction.

\section{Results and Discussion}

The Polycomb group gene MEA regulates its own imprinted expression

The $M E A$ locus is regulated by genomic imprinting where only the maternal allele is actively transcribed after fertilization. Strict maternal expression has been found for the first $52 \mathrm{~h}$ after pollination (hap) (VielleCalzada et al. 1999), but biallelic expression in the embryo was reported for later stages of seed development (Kinoshita et al. 1999). Afterward, it was discovered that paternal alleles of most genes are not expressed at detectable levels until $\geq 68$ hap (Vielle-Calzada et al. 2000). Thus, the question arose whether the allele-specific expression of $M E A$ reflected that of an average gene or whether the paternal $M E A^{p}$ allele was persistently down-regulated. For these reasons, we developed a quantitative, allele-specific assay allowing the precise detection of transcripts derived from wild-type $M E A$ and mutant mea alleles inherited either maternally or paternally (Fig. 1; Supplemental Material). Maternally derived $M E A$ transcripts accumulated to their highest level before fertilization and gradually decreased afterward (Fig. 1A,B). During that time, however, no paternal transcript was detectable (Fig. 1A,B). At later seed stages, levels of the maternal allele dropped and were barely detectable above background, while paternal transcripts remained below detection level (data not shown). Our sensitive quantitative assay thus confirmed monoallelic expression of the imprinted MEA gene throughout seed development.

Strikingly, our study revealed that mutant mea embryo sacs accumulated 45 times more maternal transcripts than wild-type before fertilization (BF) (Fig. 1B), and it persisted after fertilization (7.5 times more transcripts $4 \mathrm{~d}$ after pollination [dap]) (Fig. 1B). These findings suggest that transcription of the maternal mea allele was derepressed in mea mutant embryo sacs and seeds derived thereof. To exclude a possible cis effect influenced by the $D s$ transposon present in the mea-2 allele (Grossniklaus et al. 1998), we analyzed the expression of

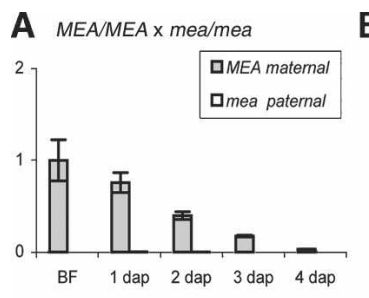

B mea/mea $\mathrm{MEA} M \mathrm{MEA}$
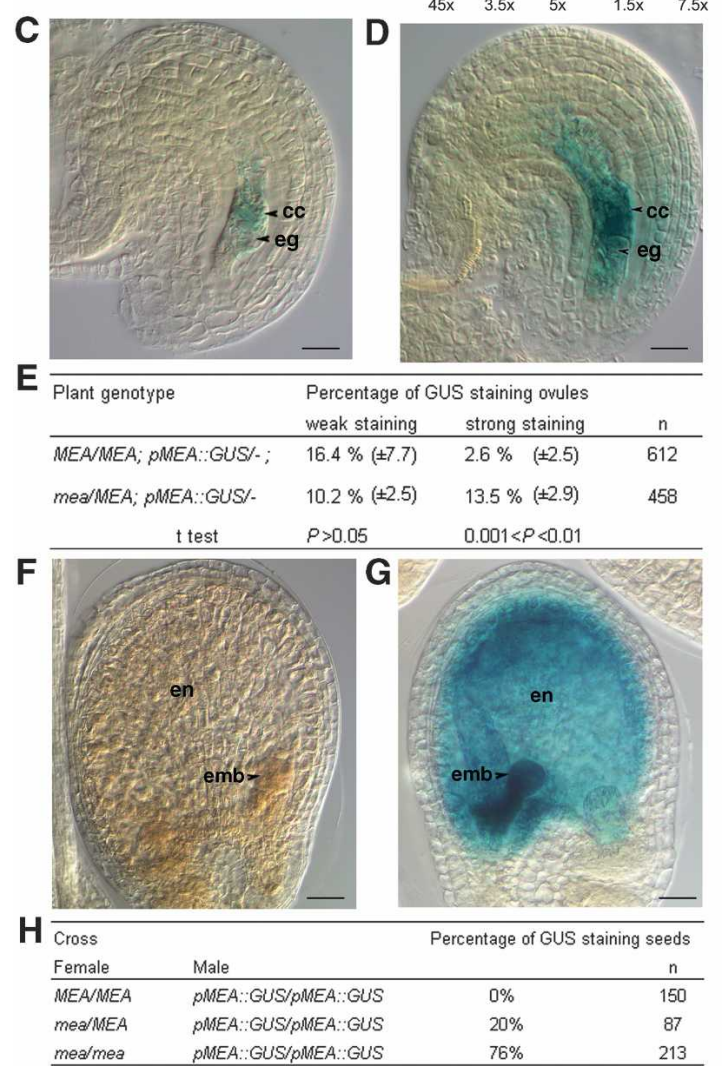

Figure 1. MEA regulates its own imprinted expression. $(A, B)$ Quantification of maternal and paternal transcripts in ovules and developing seeds derived from wild-type $(A)$ or mea-2 mutant $(B)$ embryo sacs. Transcripts were quantified before fertilization in entire gynoecia containing unfertilized ovules $(\mathrm{BF})$ and after fertilization in developing fruits following hand pollination (1-4 dap) (Supplementary Fig. S1). Transcript levels were normalized to ACTIN11; average and standard deviation of triplicate quantification are shown. In $B$, the fold increase of maternal transcripts in mea ovules compared with wild-type is indicated for each time point below the graph. $(C-E)$ Expression of a $p M E A:: G U S$ reporter gene in unfertilized ovules of $M E A / M E A$ wild-type plant $(C)$ or mea-2/MEA mutant plants $(D)$. (E) The percentage of ovules showing a weak staining as in $C$ or a strong staining as in $D$ was quantified in wild-type and mea-2/MEA mutant segregants. $(P)$ Level of significance relative to the difference between the two segregants; $(n)$ number of ovules; (cc) central cell; (ec) egg cell. Bar, $10 \mu \mathrm{m}$. $(F-H)$ Expression of a paternally inherited $p M E A:: G U S$ reporter gene 5-6 d after crossing to wildtype $(F)$ or mea-2/mea-2 mutant $(G)$ plants. In $H$, the percentage showing derepression of the usually silent paternal allele in crosses with wild-type, heterozygous, and homozygous mea-2 plants is given. $(n)$ Number of seeds analyzed. Bar, $20 \mu \mathrm{m}$.

a reporter gene under the control of the $M E A$ promoter (pMEA::GUS) in a heterozygous mea mutant background. While wild-type segregants heterozygous for the transgene $(M E A / M E A ; p M E A:: G U S /-)$ showed weak reporter gene activity (Fig. 1C,E), mutant segregants (mea) MEA; pMEA::GUS/-) showed $13.5 \%$ of ovules with 
strong staining in the embryo sac (Fig. 1D,E). Thus, the high levels of maternal MEA transcripts observed in a mea mutant background and during early development are likely due to negative autoregulation in trans. In addition, at later stages (5-6 dap) the paternally introduced pMEA::GUS transgene, which is normally silent in a wild-type background (Fig. 1F) was strongly derepressed in a maternal mea mutant background (Fig. 1G,H). Thus, MEA is also required to maintain the silent state of the paternal $M E A^{p}$ allele, but only at later stages of seed development. However, this late activation of the paternal $M E A^{p}$ allele does not have any phenotypic consequences; i.e., seeds derived from mea mutant embryo sacs still abort.

Taken together, these results demonstrate that MEA is involved in the regulation of its own imprinted gene expression. The effect is not only allele-specific but also dynamically regulated during seed development. Prior to fertilization and during early seed development, only the maternal $M E A^{m}$ allele is subject to negative autoregulation but not to complete silencing. Then, later during seed development, MEA is required to maintain silencing of the paternal $M E A^{p}$ allele. This shows interesting parallels to the EZH2-EED complex, the mammalian counterpart of the MEA-FIE complex, which is required for paternal silencing of several autosomal imprinted loci (Mager et al. 2003). MEA has differential effects on the maternal $M E A^{m}$ and the paternal $M E A^{p}$ allele with respect to developmental stage and the level of repression, indicating that MEA function is dynamically regulated during reproductive development.

\section{Maternal MEA autoregulation is direct and independent of the MEA-FIE PcG complex}

To determine whether MEA directly or indirectly regulates its own expression, we carried out ChIP experiments using the $\alpha$ MEA antibody (Köhler et al. 2003b) and tested for enrichment of fragments encompassing the entire $M E A$ promoter (Fig. 2A). Given that repression of the paternal $M E A^{p}$ allele is not biologically relevant, we concentrated our analyses on the early phase of reproductive development when repression of the maternal $M E A^{m}$ allele occurs. At this stage, $M E A$ is expressed in a very small number of cells ( 200 cells per flower), such that very little target chromatin is present in the starting material, making such experiments extremely difficult and variable. Out of six independent ChIP experiments (Supplementary Fig. S2), we found enrichment of $M E A$ promoter fragments in four independent chromatin preparations. Fragments \#3, \#5, and \#6 were enriched at least twice in these ChIP experiments (Fig. 2B). These results unequivocally demonstrate an interaction of MEA with its own promoter. The interaction occurs in a region of $\sim 800$ base pairs (bp) located 700 bp upstream of the transcriptional start site, but binding outside this core region is not excluded as fragments \#1 and \#9 were also enriched but only once (data not shown).

The finding that MEA directly autorepresses its own transcription prior to fertilization prompted us to determine whether this function was mediated by the same MEA-FIE complex that represses PHE1 expression during seed development (Köhler et al. 2003b). Clearly, the level of MEA transcripts in fis2, fie, and msi1 loss-offunction mutants, affecting the other known components of the MEA-FIE complex, was similar to the level

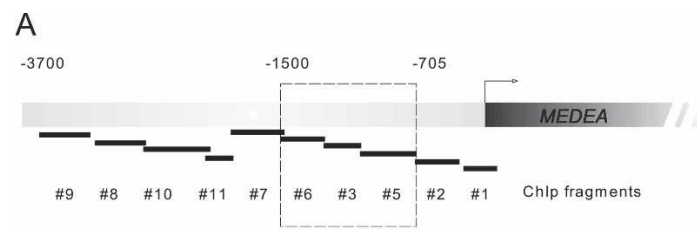

B

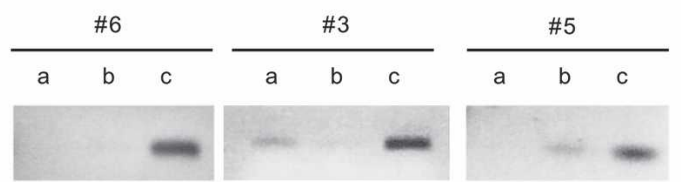

Figure 2. ChIP experiments show an interaction of MEDEA with its own promoter $(A)$ Schematic representation of the $M E A$ locus indicating the regions analyzed by PCR after ChIP. The numbers indicate a genomic position relative to the transcriptional start site (arrow) upstream the of the ORF of MEA (dark shaded gray). The dashed-line box indicates the promoter region where fragments were enriched in the immunoprecipitated fractions in the presence of the aMEA antibody. (B) ChIP experiment showing MEA interaction with its own promoter. Chromatin was prepared from $1 \mathrm{~g}$ of tissue typically consisting of $\sim 1000$ wild-type flowers. The gels show PCR products from immunoprecipitated fractions treated with no antibody (lane $a$ ), with preimmune serum (lane $b$ ), and with $\alpha$ MEA antibody (lane $c$ ). The complete set of ChIP experiments is shown in Supplementary Figure S2.

in wild-type plants before fertilization and early during seed development (Table 1). We conclude that MEA negative autoregulation around fertilization is a function that is independent of the MEA-FIE complex.

However, additional factors might be involved in $M E A$ autoregulation. Before fertilization, the level of wildtype $M E A$ transcripts in mea heterozygous plants was nine times higher than in wild-type plants (Table 1). Because $M E A$ shows gametophytic inheritance and expression (Vielle-Calzada et al. 1999), these transcripts are expected to originate from wild-type embryo sacs and not from the heterozygous $M E A /$ mea surrounding maternal tissues. This suggests that in mea heterozygous plants, $M E A$ is up-regulated in embryo sacs carrying a $M E A$ allele (50\% of the ovules). It is possible that $M E A$ has a function prior to meiosis, although expression is not detectable at this stage of development (Vielle-Calzada et al. 1999). In this case, embryo sacs carrying a wild-type genome would inherit a cytoplasm depleted in a factor normally involved in repressing $M E A$. Depletion of this factor might in turn result from haplo-insufficiency of $M E A$ prior to meiosis. Alternatively, mea may have a subtle effect in heterozygotes that leads to the accumulation of epigenetic changes, which may affect regulators of $M E A$ expression. Such secondary epigenetic effects have been observed for mutations in the DECREASE IN DNA MEHTHYLATION1 (DDM1) gene (Kakutani et al. 1996). Consistent with this hypothesis, derepression of $M E A$ is even stronger in homozygous mea mutant inbred lines (Supplementary Table S1). Finally, the Ds transposon might cause incomplete pairing at meiosis. Similar to meiotic silencing of unpaired DNA in Neurospora crassa (Shiu et al. 2001), unpaired DNA at the MEA locus could trigger an epigenetic modification rendering the $M E A^{m}$ allele in wild-type embryo sacs insensitive to autorepression.

Taken together, we provide evidence that MEA directly represses its own imprinted expression around fer- 
Baroux et al.

Table 1. Auto- and cross-regulation among the PcG genes MEA, FIS2, FIE, and MSI1

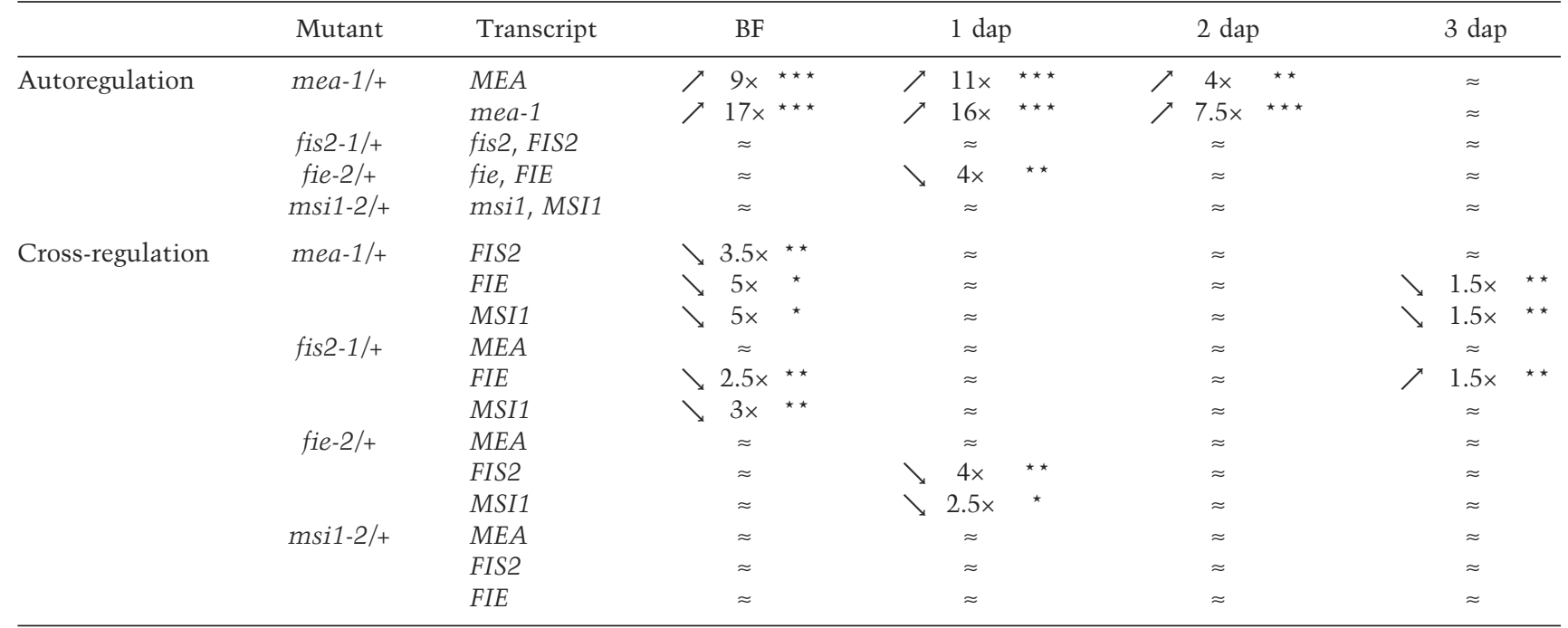

Transcript levels were measured in at least three biological replicates (independent sample collection and cDNA preparation) (Supplementary Table S1) from emasculated gynoecia (BF) or young siliques (1-3 dap) using quantitative real-time RT-PCR assays. The assay did not discriminate between transcripts derived from wild-type and mutant alleles, except for mea-1 and $M E A$, which were detected using two specific assays. Relative transcript levels were normalized to the levels of $A C T 11$. The fold increase $(\nearrow)$ or decrease $(\searrow)$ compared with wild-type levels is indicated; the level of significance was assessed using a $t$-test: $\left(^{\star}\right) 0.01<P<0.05 ;\left(^{\star \star}\right) 0.001<P<0.01$; $\left({ }^{\star \star *}\right) P<0.001 ;(\approx) P>0.05$, no significant difference. The values of transcript levels relative to wild-type levels are provided in Supplementary Table S1.

tilization. Transcriptional repression is mediated by an interaction of MEA with its own promoter and, importantly, does not require the MEA-FIE complex. All known PcG complexes in Arabidopsis involve FIE, the homolog of ESC. FIE is encoded by a unique gene and serves as platform for complex assembly (Köhler and Grossniklaus 2002; Reyes and Grossniklaus 2003), such that in fie mutants no PRC2-like complexes are formed. Thus, the autoregulatory function of MEA must involve novel, possibly non-PcG, partners that remain to be identified.

Auto- and cross-regulation among the PcG genes MEA, FIS2, FIE, and MSI1

To gain insight into potential regulatory interactions among members of the MEA-FIE complex, we investigated their expression profiles around fertilization in wild-type and loss-of-function mutants using quantitative RT-PCR.

In wild-type ovules MEA, FIS2, and MSI1 transcripts are accumulated at their highest level before fertilization and gradually decrease after fertilization, while FIE transcript levels increase transiently just after fertilization (Fig. 3A). The relative abundance of transcripts varies drastically between the FIS genes (Fig. 3B) and might be explained, in part, by their different expression patterns in ovules: Only MEA and FIS2 are specific to the embryo sac and the two fertilization products, while FIE and MSI1 expression extends to some maternal tissues (Grossniklaus et al. 1998; Vielle-Calzada et al. 1999; Luo et al. 2000; Spillane et al. 2000; Köhler et al. 2003a).

In contrast, transcript levels of the FIS genes were drastically modified in mea, fis2, and fie but not msi1 mutants, revealing both auto- and cross-regulatory feedback loops (Table 1). While fis2 and msi1 mutations had no effects on their own expression, the fie mutation

caused a severe reduction of its own transcript levels after fertilization, indicating direct or indirect autoregulation (Table 1). Before fertilization, a significant 3.5-fold to fivefold reduction of FIS2, FIE, and MSI1 transcripts was observed in mea heterozygous plants, and a similar reduction of FIE and MSI1 was found in the fis2 mutant. The reduction did not persist after fertilization, and transcripts levels were close to wild-type in mea and fis2 heterozygotes at 1 dap. By contrast, in the fie mutant FIS2 and MSI1 levels were not altered before fertiliza-

A
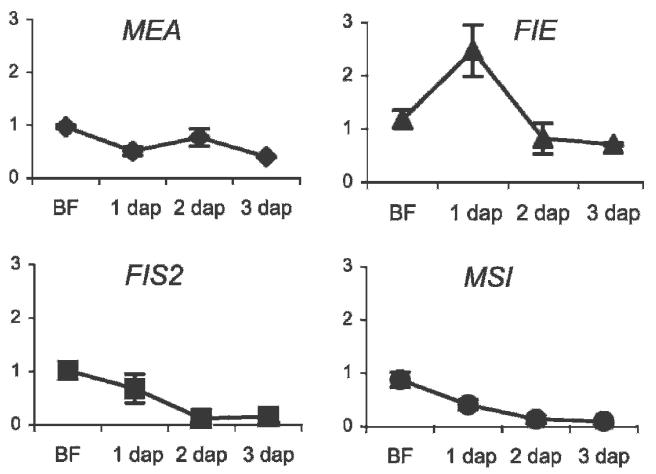

B Relative transcript abundance (BF)

\begin{tabular}{ll}
\hline MEA & 0.01 \\
FIS2 & 1 \\
FIE & 5 \\
MSI & 66 \\
\hline
\end{tabular}

Figure 3. Expression profiles of MEA, FIS2, FIE, and MSI1. (A) Relative transcript levels in wild-type gynoecia (BF) or developing siliques $(1,2$, and 3 dap). The levels were normalized to 1 before fertilization. The average of three biological replicates is plotted on the graph, and the standard deviation is shown.(B) Relative transcript abundance at the BF time point. The ratios are expressed relative to the level of FIS2, which was normalized to 1. 
tion, whereas a significant 2.5-fold and fourfold decrease was observed just after fertilization, respectively. In all mutants the levels of FIS2, FIE, and MSI1 transcripts were back to normal at 2 dap. Moderate but distinct effects on FIE and MSI1 expression levels were observed again in mea and fis 2 heterozygotes at 3 dap.

Down-regulation in fis mutants could be due to an excess-of-silencing mediated by mutant forms of PcG proteins, as described for the Tritorax-mimick mutant allele of $E(z)$ in Drosophila (LaJeunesse and Shearn 1996; Bajusz et al. 2001). This seems unlikely, however, because the lesions in the mea-1, fis2-1, and fie-2 alleles used in this study (Grossniklaus et al. 1998; Luo et al. 1999; Ohad et al. 1999/ cause truncation in the SET, VEFS, and WD40 domains of MEA, FIS2, and FIE, respectively, which are essential to the formation of a functional PcG complex (Denisenko et al. 1998; Cao and Zhang 2004; Chanvivattana et al. 2004; Yamamoto et al. 2004; Nekrasov et al. 2005). Instead, it is likely that $M E A, F I S 2$, and FIE directly or indirectly activate FIS2, FIE, and MSI1 expression.

Our results clearly show that expression of the FIS genes is modulated around fertilization by auto- and cross-regulatory interactions (Fig. 4). Interestingly, FIE autoregulation and MEA positive regulation of FIE exactly reflects the situation in Drosophila, where ESC autoregulates and $E(Z)$ positively regulates ESC (Ali and Bender 2004). Positive cross-regulation extends to other members of the MEA-FIE complex, except MSI1, which does not influence MEA, FIS2, and FIE expression, although these proteins in turn regulate MSI1 expression.

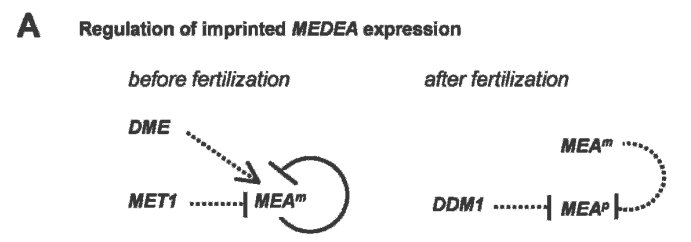

B

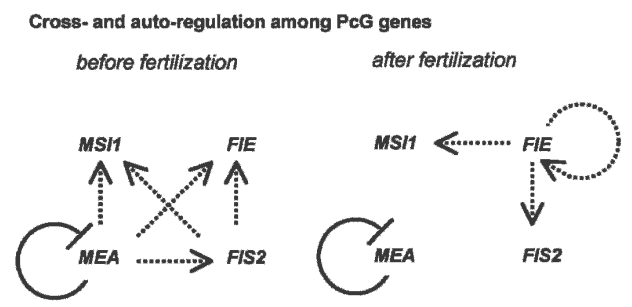

Figure 4. Complex regulatory interactions among the FIS class PcG genes. (A) MEA regulates its own imprinted gene expression. Schematic representation of the known regulators of imprinted $M E A$ expression. Direct interactions are shown as solid lines, and interactions that are either direct or indirect are shown as stippled lines. Before fertilization, the maternal $M E A^{m}$ allele is activated through antagonistic interactions of DME and MET1 (Xiao et al. 2003) and kept at a low expression level by direct autorepression. After fertilization, the paternal $M E A^{p}$ allele is maintained silent through $M E A$-mediated repression and activity of the chromatin remodeling factor DDM1, based on genetic evidence (Vielle-Calzada et al. 1999). (B) Genetic regulation of the FIS class PcG genes involves positive auto- and cross-regulations. Only MEA and FIE autoregulate. MEA autorepression is direct but independent of the other components of the MEA-FIE complex. The mechanism of FIE autoregulation is unknown but is also independent of the MEA-FIE complex. A comparison of regulatory interactions before and after fertilization illustrates the highly dynamic nature of PcG regulation.
The physiological significance of these complex regulatory interactions for seed development remains to be elucidated. Most likely they ensure the fine-tuning of the MEA-FIE complex protein levels and may regulate their dynamic participation in distinct variants or subcomplexes during development.

The positive feedback loops in this cross-regulatory network are noteworthy (Fig. 4). Two possibilities arise to explain the activating function of MEA, FIS2, and FIE. Possibly, they could down-regulate the expression of transcriptional repressors of FIS2, FIE, and MSI1. Alternatively, they could take part in novel activating complexes. The latter hypothesis is based on antagonistic repression/activation properties reported for several PcG proteins in Drosophila. E(Z) and other PcG genes have been reclassified as Enhancers of trithorax and Polycomb (ETP) on the basis that some mutant alleles could not only enhance Polycomb but also trithorax homeotic defects (LaJeunesse and Shearn 1996; Gildea et al. 2000; Bajusz et al. 2001). Thus, MEA, FIS2, and FIE may be plant ETPs that, as Drosophila ETPs, take part in distinct complexes with transcriptional repression and activation properties (Brock and van Lohuizen 2001).

In summary, our studies have not only identified $M E A$ as a novel direct regulator of its own imprinted gene expression, but have also highlighted the complex and highly dynamic nature of auto-and cross-regulatory interactions among the FIS genes. Clearly, MEA has distinct effects on gene regulation at various stages of reproductive development, likely reflecting a dynamic composition of MEA-containing complexes. This dynamic behavior may indicate that, in plants, PcG repression is not as persistent as it is in animals but is often released upon external or internal stimuli, such as fertilization.

\section{Material and methods}

\section{Plant material}

Wild-type plants were Arabidopsis thaliana (L.) Heynh. accession Landsberg (erecta mutant, Ler) and accession C24. The mutant alleles used were mea-1, mea-2 (Grossniklaus et al. 1998), fis2-1 (Luo et al. 1999), fie-2 (Ohad et al. 1999), and msi1-2 (Guitton et al. 2004). The mea-1 and mea-2 mutant plants were selected on kanamycin, while the others were genotyped using SSLP or dCAPs markers (Supplemental Material). Establishment of the $p M E A:: G U S$ transgenic lines and staining procedures are described in Supplemental Material.

\section{Quantitative RT-PCR}

The accumulation of MEA, mea, FIS2, FIE, and MSI1 transcripts were measured using quantitative real-time RT-PCR as described by Köhler et al. (2003b). Details can be found in the Supplemental Material and Supplementary Figure S3. In brief, quantitative analyses of transcript levels were carried out using Sybr Green or Taqman real-time PCR assays (Applied Biosystem). Three PCR replicates were performed for each cDNA sample, and the specificity and amount of the unique amplification product were determined according to the manufacturer's instructions (Applied Biosystems). For measuring maternal and paternal MEA transcripts, we used the mea-1 and mea-2 mutant alleles (Grossniklaus et al. 1998), which produce mutant transcripts containing transposon sequences (Supplementary Fig. S3), allowing specific detection and discrimination from wild-type MEA transcripts. We applied this assay to distinguish maternal and paternal transcripts in a set of reciprocal crosses between wild-type and homozygous mea-2 (Fig. 1) or in selfed mea-1 heterozygous lines (Table 1). In all experiments, transcript levels were normalized to the level of ACTIN11, which is expressed in the gametophyte and zygotic products of the seed (embryo and endosperm) but not in the surrounding maternal tissues (Huang et al. 1997). Beyond 4 dap, ACT11 levels decrease and cannot be used for normalization (data not shown). 
ChIP and PCR

Six independent experiments were carried out using 1000 flowers and young siliques around fertilization ( $1 \mathrm{~g}$ tissue). We estimated that the starting material encloses $\sim 50,000$ female gametophytes (where MEA is expressed) containing 50-150 fg target DNA (for a 1-kb fragment) before immunoprecipitation. ChIP was performed according to Köhler et al. (2003b) with one minor modification: Reverse cross-linking was carried out overnight at $65^{\circ} \mathrm{C}$.

\section{Acknowledgments}

We thank Claudia Köhler for providing affinity-purified $\alpha$ MEA antibody, Stéphane Pien for technical advice, and Anja Schmidt and Hans-Peter Schöb for critical reading of the manuscript. This work was supported by the University of Zürich and a grant from the Swiss National Science Foundation (to U.G.).

\section{Note added in proof}

Recently, two other publications reported regulation of the paternal $M E A^{p}$ allele by the MEA-FIE complex and showed that paternal silencing is correlated with histone methylation on H3K27 (Gehring et al. 2006; Jullien et al. 2006).

\section{References}

Ali, J.Y. and Bender, W. 2004. Cross-regulation among the polycomb group genes in Drosophila melanogaster. Mol. Cell. Biol. 24: $7737-$ 7747.

Bajusz, I., Sipos, L., Gyorgypal, Z., Carrington, E.A., Jones, R.S., Gausz, J., and Gyurkovics, H. 2001. The Trithorax-mimic allele of Enhancer of zeste renders active domains of target genes accessible to polycombgroup-dependent silencing in Drosophila melanogaster. Genetics 159: $1135-1150$.

Brock, H.W. and van Lohuizen, M. 2001. The Polycomb group: No longer an exclusive club? Curr. Opin. Genet. Dev. 11: 175-181.

Cao, R. and Zhang, Y. 2004. The functions of E(Z)/EZH2-mediated methylation of lysine 27 in histone H3. Curr. Opin. Genet. Dev. 14: 155-164.

Chanvivattana, Y., Bishopp, A., Schubert, D., Stock, C., Moon, Y.H., Sung, Z.R., and Goodrich, J. 2004. Interaction of Polycomb-group proteins controlling flowering in Arabidopsis. Development 131: 5263-5276.

Choi, Y., Harada, J.J., Goldberg, R.B., and Fischer, R.L. 2004. An invariant aspartic acid in the DNA glycosylase domain of DEMETER is necessary for transcriptional activation of the imprinted MEDEA gene. Proc. Natl. Acad. Sci. 101: 7481-7486.

Denisenko, O., Shnyreva, M., Suzuki, H., and Bomsztyk, K. 1998. Point mutations in the WD40 domain of Eed block its interaction with Ezh2. Mol. Cell. Biol. 18: 5634-5642.

Gehring, M., Huh, J.H., Hsieh, T.F., Penterman, J., Choi, Y., Harada, J.J., Goldberg, R.B., and Fischer, R.L. 2006. DEMETER DNA glycosylase establishes MEDEA polycomb gene self-imprinting by allele-specific demethylation. Cell 124: 495-506.

Gildea, J.J., Lopez, R., and Shearn, A. 2000. A screen for new trithorax group genes identified little imaginal discs, the Drosophila melanogaster homologue of human retinoblastoma binding protein 2. Genetics 156: 645-663.

Grossniklaus, U., Vielle-Calzada, J.P., Hoeppner, M.A., and Gagliano, W.B. 1998. Maternal control of embryogenesis by MEDEA, a polycomb group gene in Arabidopsis. Science 280: 446-450.

Guitton, A.E. and Berger, F. 2005. Control of reproduction by Polycomb Group complexes in animals and plants. Int. J. Dev. Biol. 49: 707716.

Guitton, A.E., Page, D.R., Chambrier, P., Lionnet, C., Faure, J.E., Grossniklaus, U., and Berger, F. 2004. Identification of new members of Fertilisation Independent Seed Polycomb Group pathway involved in the control of seed development in Arabidopsis thaliana. Development 131: 2971-2981.

Hennig, L., Taranto, P., Walser, M., Schonrock, N., and Gruissem, W. 2003. Arabidopsis MSIl is required for epigenetic maintenance of reproductive development. Development 130: 2555-2565.

Huang, S., An, Y.Q., McDowell, J.M., McKinney, E.C., and Meagher, R.B. 1997. The Arabidopsis ACT11 actin gene is strongly expressed in tissues of the emerging inflorescence, pollen, and developing ovules.
Plant Mol. Biol. 33: 125-139.

Jullien, P.E., Katz, A., Oliva, M., Ohad N., and Berger, F. 2006. Polycomb group complexes self-regulate imprinting of the Polycomb group gene MEDEA in Arabidopsis. Curr. Biol. 16: 486-492.

Kakutani, T., Jeddeloh, J.A., Flowers, S.K., Munakata, K., Richards, E.J. 1996. Developmental abnormalities and epimutations associated with DNA hypomethylation mutations. Proc. Natl. Acad. Sci. 93: 12406-12411.

Kinoshita, T., Yadegari, R., Harada, J.J., Goldberg, R.B., and Fischer, R.L. 1999. Imprinting of the MEDEA polycomb gene in the Arabidopsis endosperm. Plant Cell 11: 1945-1952.

Köhler, C. and Grossniklaus, U. 2002. Epigenetic inheritance of expression states in plant development: The role of Polycomb group proteins. Curr. Opin. Cell Biol. 14: 773-779.

Köhler, C., Hennig, L., Bouveret, R., Gheyselinck, J., Grossniklaus, U., and Gruissem, W. 2003a. Arabidopsis MSIl is a component of the MEA/FIE Polycomb group complex and required for seed development. EMBO J. 22: 4804-4814.

Köhler, C., Hennig, L., Spillane, C., Pien, S., Gruissem, W., and Grossniklaus, U. 2003b. The Polycomb-group protein MEDEA regulates seed development by controlling expression of the MADS-box gene PHERES1. Genes \& Dev. 17: 1540-1553.

LaJeunesse, D. and Shearn, A. 1996. E(z): A Polycomb group gene or a trithorax group gene? Development 122: 2189-2197.

Luo, M., Bilodeau, P., Koltunow, A., Dennis, E.S., Peacock, W.J., and Chaudhury, A.M. 1999. Genes controlling fertilization-independent seed development in Arabidopsis thaliana. Proc. Natl. Acad. Sci. 96: 296-301.

Luo, M., Bilodeau, P., Dennis, E.S., Peacock, W.J., and Chaudhury, A. 2000. Expression and parent-of-origin effects for FIS2, MEA, and FIE in the endosperm and embryo of developing Arabidopsis seeds. Proc. Nat1. Acad. Sci. 97: 10637-10642.

Mager, J., Montgomery, N.D., de Villena, F.P., and Magnuson, T. 2003. Genome imprinting regulated by the mouse Polycomb group protein Eed. Nat. Genet. 33: 502-507.

Nekrasov, M., Wild, B., and Muller, J. 2005. Nucleosome binding and histone methyltransferase activity of Drosophila PRC2. EMBO Rep. 6: 348-353.

Ohad, N., Yadegari, R., Margossian, L., Hannon, M., Michaeli, D., Harada, J.J., Goldberg, R.B., and Fischer, R.L. 1999. Mutations in FIE, a WD Polycomb group gene, allow endosperm development without fertilization. Plant Cell 11: 407-416.

Otte, A.P. and Kwaks, T.H. 2003. Gene repression by Polycomb group protein complexes: A distinct complex for every occasion? Curr. Opin. Genet. Dev. 13: 448-454.

Reyes, J.C. and Grossniklaus, U. 2003. Diverse functions of Polycomb group proteins during plant development. Semin. Cell Dev. Biol. 14: $77-84$.

Ringrose, L. and Paro, R. 2004. Epigenetic regulation of cellular memory by the Polycomb and Trithorax group proteins. Annu. Rev. Genet. 38: 413-443.

Shiu, P.K., Raju, N.B., Zickler, D., and Metzenberg, R.L. 2001. Meiotic silencing by unpaired DNA. Cell 107: 905-916.

Spillane, C., MacDougall, C., Stock, C., Köhler, C., Vielle-Calzada, J.P., Nunes, S.M., Grossniklaus, U., and Goodrich, J. 2000. Interaction of the Arabidopsis Polycomb group proteins FIE and MEA mediates their common phenotypes. Curr. Biol. 10: 1535-1538.

Valk-Lingbeek, M.E., Bruggeman, S.W., and van Lohuizen, M. 2004. Stem cells and cancer; the Polycomb connection. Cell 118: 409-418.

Vielle-Calzada, J.P., Thomas, J., Spillane, C., Coluccio, A., Hoeppner, M.A., and Grossniklaus, U. 1999. Maintenance of genomic imprinting at the Arabidopsis medea locus requires zygotic DDM1 activity. Genes \& Dev. 13: 2971-2982.

Vielle-Calzada, J.P., Baskar, R., and Grossniklaus, U. 2000. Delayed activation of the paternal genome during seed development. Nature 404: 91-94.

Xiao, W., Gehring, M., Choi, Y., Margossian, L., Pu, H., Harada, J.J., Goldberg, R.B., Pennell, R.I., and Fischer, R.L. 2003. Imprinting of the MEA Polycomb gene is controlled by antagonism between MET1 methyltransferase and DME glycosylase. Dev. Cell 5: 891-901.

Yamamoto, K., Sonoda, M., Inokuchi, J., Shirasawa, S., and Sasazuki, T. 2004. Polycomb group suppressor of zeste 12 links heterochromatin protein $1 \alpha$ and enhancer of zeste 2. J. Biol. Chem. 279: 401-406. 